\title{
Structural optimization of a small aircraft wing from aeroelastic responses
}

\author{
L. Fitschy, F. Thirifay, E. Wyart, S. Pierret \& P. Geuzaine \\ CENAERO, Gosselies, Belgium
}

\begin{abstract}
The objective of weight reduction and the introduction of new materials lead to aeronautical structures that are less stiff. As a consequence, coupled fluid-structure simulations become essential to predict the performance of aircraft. In addition, structural design can now benefit from the use of mature optimization techniques. In this paper, these two disciplines are combined and applied to the structural design of a lightweight six-passenger aircraft wing.

Keywords: aeroelasticity, optimization, structural design, code coupling.
\end{abstract}

\section{Introduction}

A large class of fluid-structure interaction problems require the simultaneous application of computational fluid dynamics (CFD) and computational structural dynamics (CSD). Since each discipline has developed powerful specialized tools, a partitioned procedure is preferred for solving coupled field nonlinear fluid-structure interactions (FSI) problems. During the past years, CENAERO has developed a FSI platform involving the coupling of its three-dimensional unstructured flow solver Argo with the Samcef Mecano [1] finite element structural solver through the $\mathrm{MpCCI}$ [2] software in order to perform aeroelastic simulations. CENAERO has also developed an optimization software Max to deal with complex optimization problems. This software allows to perform derivative free optimization with very few calls to computer intensive simulation software. The method is based on the use of a genetic algorithm using real coded variables and on the construction of an approximate model.

In this work, the FSI platform and the optimization software are coupled to perform the optimization of the structural model of a lightweight six-passenger aircraft wing with multi-disciplinary constraints. The objective is to minimize 
the weight of the structure and to guarantee a safety lift accordingly to the JAR norm. Additional constraints are imposed on the maximal elastic limit allowed by the material and the maximum aeroelastic deformation of the wing. The optimization procedure finds simultaneously the optimal sections of the stringers and the thicknesses of the webs, the ribs and the skin elements.

To address the topics outlined above, the remainder of this paper is organized as follows. In the following section, the FSI platform is described. The optimization platform is reviewed in Sec. 3. The potential of the proposed approach is illustrated and evaluated with the optimization of the wing structure of a six-passenger aircraft in Sec. 4. Finally, concluding remarks are offered.

\section{FSI platform}

\subsection{CFD solver}

The CFD solver Argo developed at CENAERO is a domain decomposition-based parallel 3D Navier-Stokes solver which uses a hybrid finite volume and finite element discretization on unstructured tetrahedral meshes [3, 4]. Pseudo timeintegration is performed for steady state flows with the backward Euler scheme. Since this scheme is implicit, a system of nonlinear equations must be solved at each time-step. For this purpose, an inexact Newton method based on a finite difference Newton-GMRES algorithm [5, 6] is used.

\subsection{CSD solver}

Samcef [1] is a general-purpose finite element commercial software for structural and heat transfer analysis. Its applications cover mechanical, electrical, aeronautical, nuclear and naval engineering industries as well as the fields of transport, civil and offshore engineering. In the present work, the Mecano Structure module of Samcef is used. This module is dedicated to static, quasi-static and dynamic analysis of nonlinear structures. It is also possible to impose a purely linear geometric behavior without any second order effects. This module is chosen because it provides access to user-defined functions that ease the coupling with the $\mathrm{MpCCI}$ library. Note that in the present work the structure is represented by a finite element model, and its dynamic behavior is predicted by using the displacement, velocity, and acceleration degrees of freedom rather than a modal representation.

\subsection{Coupled FSI solution}

The CFD and CSD solvers are coupled through the MpCCI (Mesh based parallel Code Coupling Interface) software [2] in order to perform aeroelastic simulations. $\mathrm{MpCCI}$ is a software environment that provides an application independent interface for coupling different simulation codes. It enables the exchange of data (e.g. displacement, force) between the fluid and structure meshes along the fluidstructure interface. It offers linear and bilinear, conservative and non-conservative 
interpolations when these meshes are not compatible, which is usually the case for aeroelastic applications.

Although MpCCI supports purely MPI-based communications with one common MPI world communicator for all coupled codes, the communication scheme based on a client-server concept is adopted in this work because it allows to separate the codes into their own independent communication worlds. This has the advantage that each code uses its own MPI (or any other communication library) for its internal parallelization. There are therefore no restrictions or interferences with the communication library used by the remote codes. In this client-server approach the codes are started independently in the same way as in the standalone case. The coupling is done via additional "coupling server" processes (one for each code process) which live in a common MPI world and communicate with each code process through low-level interprocess communication (e.g. sockets).

The various modules of the MpCCI environment have to be linked to and called by the simulation codes. This linkage is trivial for any in-house code, as the CFD solver in this work. The connection to a commercial code, like Samcef Mecano, can be performed through its user programming interface.

An iterative partitioned procedure is used to solve steady and unsteady nonlinear aeroelastic problems. A generic cycle of this procedure can be described as follows: (1) transfer a prediction of the displacement of the wet boundary of the structure to the fluid subsystem and update the position of the fluid mesh (with a structure analogy method) accordingly, (2) advance the fluid subsystem, (3) compute the fluid force and transfer it to the structural code, (4) advance the structure subsystem.

In the steady case, the above four-step procedure relies on the backward Euler scheme for advancing the fluid subsystem and a quasi-steady algorithm for advancing the structure subsystem. In order to avoid strong variations of the fluid-structure interface between two successive iterations, an under-relaxation factor can be used for the transfer of the displacement of the wet boundary of the structure.

\section{Optimization platform}

\subsection{Optimization solver}

The optimization solver Max developed at CENAERO is based on the use of genetic algorithms (GA) which provide a very robust method. One drawback of GAs is that they suffer from a slow convergence because they use probabilistic recombination operators to control the step size and searching direction. As a consequence, for real industrial problems involving expensive function evaluations, the GA-required computational time is usually impractical even with today's computing power. To improve this, the optimization process is accelerated by the use of an approximate model based on neuronal network. 


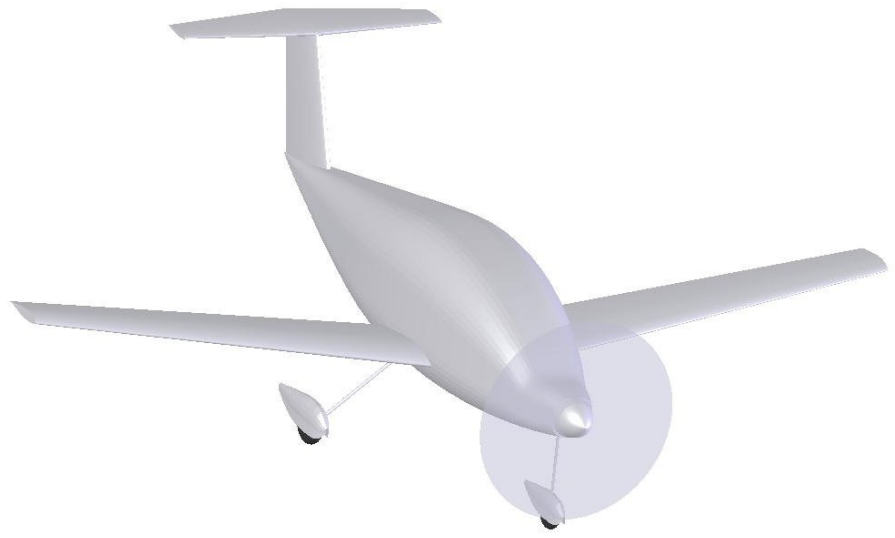

Figure 1: CAD representation of the six-passenger aircraft.

\subsection{FSI-based optimization}

One great advantage of GAs is that they do not need any derivative calculation to achieve their task. Only objective functions and constrains values are required. Thus, the FSI platform can be integrated in the process without major difficulties provided that the required values are correctly exported in particular files.

\section{Application to the design of a small aircraft wing}

In order to highlight the potential of the methodology presented in this paper, the optimization of the structural design of a small aircraft wing is considered. This six-passenger aircraft (see Fig. 1) is approximatively $8 \mathrm{~m}$ long and its cruise fly condition is Mach 0.4 at an altitude of $3500 \mathrm{~m}$. The wing is slightly twisted $\left(3^{\circ}\right.$ between wing root and wing tip), has an aspect ratio of 10 and a span of $11 \mathrm{~m}$. The wing section in the streamwise direction is made of a NLF(1)-0416 airfoil.

\subsection{CFD and CSD models}

The CFD model is composed of the wing and the fuselage of the aircraft. Other components are neglected for this optimization due to their assumed minor influence on the flow around the wing. Since symmetrical flow conditions are considered, only half of the geometry is modeled. Only inviscid steady simulations with an ideal gas are considered at this design stage. A sensitivity study has been performed and has shown that the best ratio between computational time and accuracy was obtained with a mesh of approximatively 1 million tetrahedra. Figure 2(a) displays the wing surface mesh.

From a structural point of view, the geometry is limited to the wing (see Fig. 2(b)). Since its internal structure has to be optimized, the main components of 
a real wing are modeled. These are the panels of the skin, the beams in the span direction (referred to as stringers), the vertical panels in the span direction (referred to as webs) and the vertical panels in the stream direction (referred to as ribs). The skin, webs and ribs are modeled by shell finite elements. The stringers are modeled by beam finite elements. The model is clamped at the wing root because the fuselage is assumed to be infinitely rigid. Since the wing aspect ratio is rather large and can thus induce large displacements of the wing tip, geometric nonlinearities are taken into account in the structural solver. One of the main advantage of a nonlinear structural solver is that buckling can be predicted. For the optimization phase, it is important to detect when buckling appears and notice the optimizer that this solution should be rejected. A sensitivity analysis has shown that the best compromise between computational time and accuracy was obtained with a mesh of approximatively 100,000 degrees of freedom. The structural model is entirely parameterized, thus the modification of a parameter implies the generation of a new CAD model followed by the creation of the corresponding CSD mesh.

\subsection{Aeroelastic response analysis}

The fluid-structure interface participating to the coupling is composed of the skin panels in the CSD code and the wing surfaces in the CFD code. Sample results of a steady aeroelastic computation using the FSI platform are shown in Fig. 3 for freestream conditions set to $M=0.4$ and $\alpha=-4.5^{\circ}$, which correspond to trimmed conditions at maximum speed. A steady state solution is first computed around the undeformed configuration of the wing. This solution is then used as an initial condition, and the steady aeroelastic response of the wing is computed with the procedure described in Sec. 2.3. Figure 3(a) displays the final deformed shape of the wing, while Fig. 3(b) reports the convergence of the computed aerodynamical forces.

\subsection{Aeroelastic-based optimization}

As mentioned previously, the structural model is entirely parameterized with several design variables. These include the beam square section area and the shell thickness, all function of their position in the span direction. They also include the number and thickness of the ribs and webs as well as their position with respect to the chord.

Since the use of all design parameters in the optimization process would lead to an unacceptable computational time, some preliminary aeroelastic calculations are performed without the optimization loop in order to analyze the influence of some design parameters. Different behaviors can be observed. The number of webs and the skin thickness have a large influence on the aerodynamic efficiency. An increased number of webs guarantees small rotations of the wing and thus the stability of the effective incidence. Both parameters control the airfoil section shape which is crucial to ensure good performance. An increased number of ribs maintains the cross-section shapes and also offers a way to control the tip displacement. 
178 Fluid Structure Interaction and Moving Boundary Problems IV

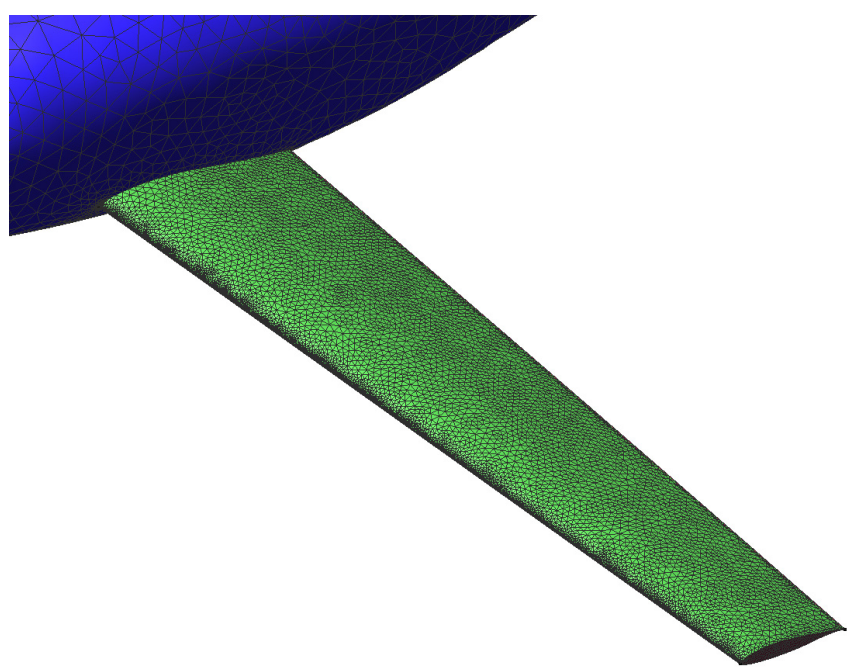

(a) CFD model (surface mesh)

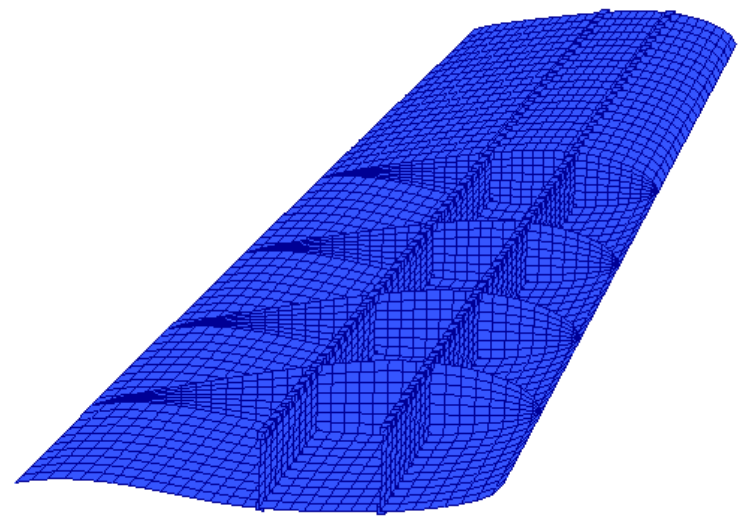

(b) CSD model

Figure 2: CFD and CSD models of the six-passenger aircraft.

The selected objective function to be minimized by the optimization solver is the weight of the wing. In order to obtain a realistic solution and to guarantee minimal aerodynamic performance the following constraints are imposed. First, an upper

WIT Transactions on The Built Environment, Vol 92, (C) 2007 WIT Press

www.witpress.com, ISSN 1743-3509 (on-line) 


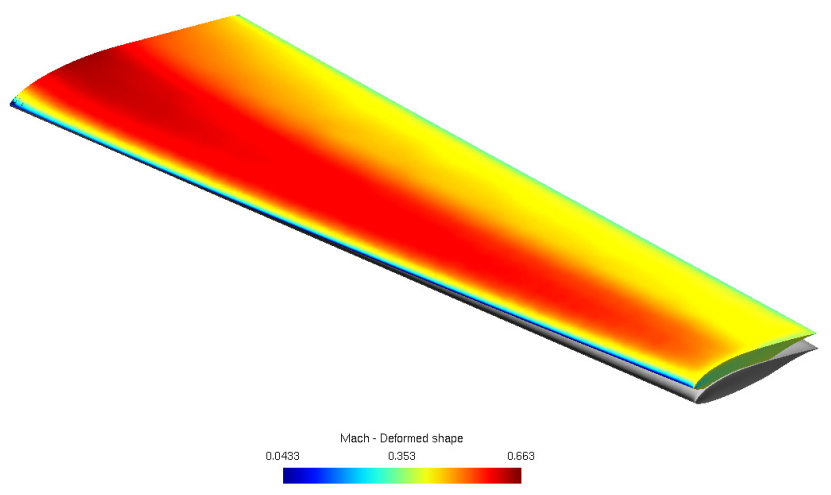

(a) Undeformed (solid) and deformed (colored with Mach number contours) shapes

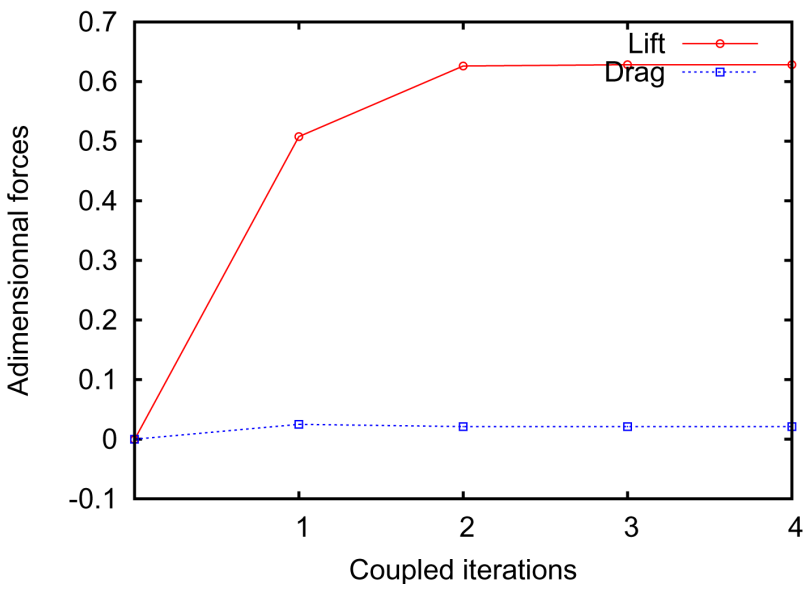

(b) Aerodynamical forces convergence

Figure 3: Steady aeroelastic response of the wing of the six-passenger aircraft.

limit on the maximal stress allowed in the material is prescribed. Second, the lift is imposed to remain larger than $95 \%$ of its trimmed value. Third, a constraint on the wing tip displacement is prescribed. Following the preliminary aeroelastic calculation results the number of beams, ribs and webs is fixed respectively to 4, 7 and 14. Furthermore their positions are also fixed in order to limit the number of parameters. The parameters considered for the optimization are thus the sections of the beams and the thicknesses of the ribs, webs and skins. This choice leads to 


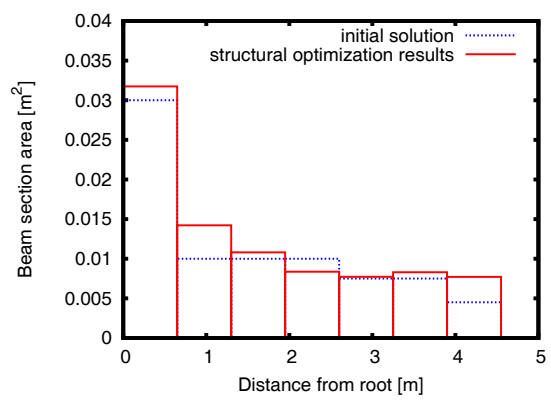

(a) Beam optimization without FSI

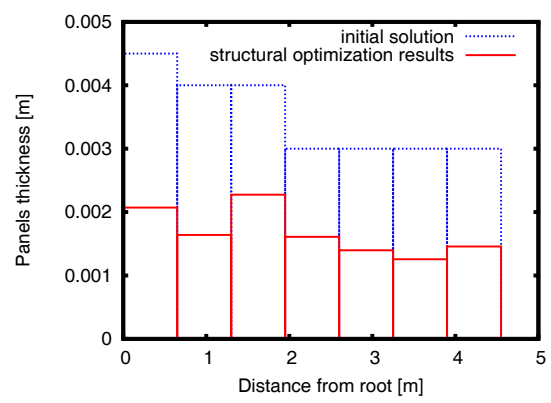

(c) Skin optimization without FSI

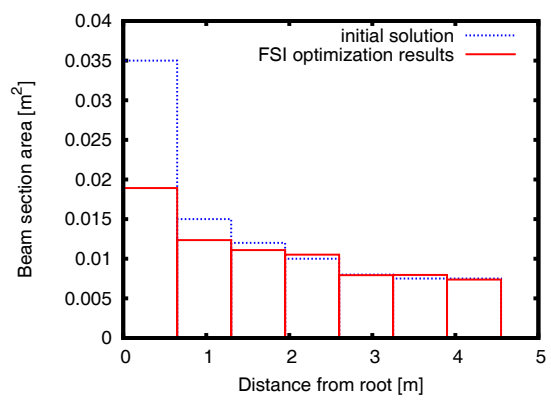

(b) Beam optimization with FSI

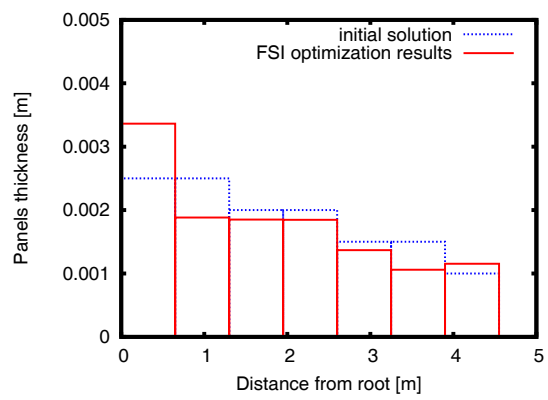

(d) Skin optimization with FSI

Figure 4: Optimized wing of the six-passenger aircraft.

a number of parameters of about 50 .

The optimization is first performed without the aeroelastic coupling — that is, the tip displacement is computed with the aerodynamic loading corresponding to the original geometry. The results are presented in Figs. 4(a) and 4(c) which show respectively the evolution of the beam section and the skin thickness. It is interesting to note that this optimized geometry buckles if the actual aerodynamic loading is considered, thus showing the significance of taken into account the aeroelastic computation. Therefore a second optimization is performed with the tip displacement computed from a steady aeroelastic simulation. The evolution of the beam section and the skin thickness resulting from this aeroelastic-based optimization are shown respectively in Figs. 4(b) and 4(d). As expected, there is a transfer of material from the beams to the skin panels. Regarding the computational cost of the optimization, about 200 structural models are evaluated to find an optimal value for the 50 design parameters. 


\section{Conclusions}

An approach to perform the structural design of lightweight aircraft wings is presented in this paper. The approach combines genetic-based optimization techniques accelerated by meta-models with steady aeroelastic three-dimensional simulations. The potential of the approach is demonstrated for the weight minimization of a six-passenger aircraft wing subjected multi-disciplinary constraints.

\section{References}

[1] SAMTECH, SAMCEF 11.1 User Manual.

[2] Wolf, K., MpCCI - a general coupling library for multidisciplinary simulation. Workshop on Scalable Solver Software Multiscale Coupling and Computational Earth Science, 2001.

[3] Fezoui, F., Lanteri, S., Larrouturou, B. \& Olivier, C., Résolution numérique des équations de Navier-Stokes pour un fluide compressible en maillage triangulaire. Technical Report 1033, INRIA, France, 1989.

[4] Barth, T.J., Numerical aspects of computing high Reynolds number flows on unstructured meshes, 1991. AIAA paper 91-0721.

[5] Brown, P.N. \& Saad, Y., Hybrid Krylov methods for nonlinear systems of equations. SIAM Journal on Scientific and Statistical Computing, 11(3), pp. 450-481, 1990.

[6] Geuzaine, P., Newton-Krylov strategy for compressible turbulent flows on unstructured meshes. AIAA Journal, 39(3), pp. 528-531, 2001. 